\title{
Analysis of Building Object Detection Methods Using Remote Sensing Images
}

\author{
Paarivallal R A ${ }^{\mathrm{a},}{ }^{1}$, Vakula $\mathrm{V}^{\mathrm{b}}$, Hemavikasini $\mathrm{S}^{\mathrm{b}}$, and Vidhya $\mathrm{S}^{\mathrm{b}}$ \\ ${ }^{a}$ Assistant professor, BannariAmman institute of technology, India \\ ${ }^{\mathrm{b}}$ PG Scholars, Bannari Amman institute of technology, India
}

\begin{abstract}
Automatic buildings detection from very high resolution (VHR) satellite images is the recent exploration hotspot in remote sensing images. In this work presents a different method to detect the buildings by atomization of the training area collecting stage aimed at supervised classification. The classification process for the exploration of satellite image built on Pixel Intensity Clustering Algorithm. This method engagements the multi-spectral remote sensing information system to discover spectral signature of different objects such as land cover classification, vegetation, rural and urban areas, road structure, concrete structure, rocky areas and remaining areas presented in the image. In the testing, the proposed SVM framework outperformed for the three (Linear SVM, Cubic SVM, Medium Gaussian SVM) methods tested. SVM is trained complete supervised learning to classify to each location in the image as "buildings" or "Nonbuildings". Finally, SVM algorithm to find the accuracy value, training time and receiver operating characteristic curves towards estimate detection performance, and equate the proposed algorithm with few existing methods.
\end{abstract}

Keywords. Remote sensing images, Otsu' segmentation, SVM Classification

\section{Introduction}

The remote sensing information consumes several application spaces including soil moisture measurement, land cover classification, and forest type classification, estimation to the fluid water substance of vegetation, hail mapping, ocean snownature characterization, and oceanography [1]. The science and abilities of gaining information and extrication the highlights in the type of otherworldly, threedimensional and sequential around certain objects, area or spectacle [3]. For example vegetation, land cover classification, urban area, agriculture land deprived of coming into physical interaction of these items [4]. In this paper, the multispectral picture of Erode district is utilized to ascertain the level of flexible highlights, for example vegetation, water bodies, land spread arrangement, solid structures, rough zone, urban regions, street construction, and outstanding zone exhibited in this image [6].The National Aeronautics and Space Administration (NASA) of USA collects a 7- band data that are used to the feature extraction. It's known as the Land Remote Sensing Satellite (LANDSAT) image [8]. The Indian National Satellite System(INSAT) picture comprises 3-band data and this data is cultivated in the assistance of these 3 bands termed near-infrared band, red band, and green band. Each band hasabout particular data and through the assistance of these three bands, the features are extricated. In view of wavelength, remote-sensing is classified mad about three sorts for example visible and reflective infrared remote sensing, thermal infrared remote sensing, and microwave remote sensing [2]. The visible red and near-infrared (NIR)

\footnotetext{
${ }^{1}$ Paarivallal R A, Assistant Professor, Bannari Amman Institute of Technology, India;

E-mail: nrdmspi@gmail.com.
} 
The classification technique is used to classify different features of the remote sensing images. There are more of classification algorithm available, for example, SVM, random forest, and neural network. In this work used to the SVM classification method [7]. The info included is a portrayal that catches the embodiment of the object under classification. SVM is trained concluded supervised learning to classify each place in the image as "buildings" or "Non-buildings." Finally, SVM algorithm to find the accuracy value, training time and roc characteristics. In this work, the study area is defined in Section 2. The proposed methodology of building detection and classification is described in Section 3. Section 4 contain implementation consequences of the proposed method and the result discussions. Finally, assumption is providing in Section 5.

\subsection{Study area}

The Sathyamangalam (Rural area) of Tamilnadu is located between $10^{\circ} 29^{\prime} 15^{\prime \prime}$ to $11^{\circ} 43^{\prime} 11^{\prime \prime} \mathrm{N}$ latitude and $76^{\circ} 50^{\prime} 46^{\prime \prime}$ to $77^{\circ} 27^{\prime} 22^{\prime \prime} \mathrm{E}$ longitude covers $1400 \mathrm{~km}^{2}$ The topography of the reserve is greatly variable with grasslands, angles, mountains and waterways. A current study is limited within the core zone area of the sathyamangalam (2272 sq.km), but this urban area is characterized by population density and buildings. This remote sensing project will start with the from google maps imagery a small region of Sathyamangalam.

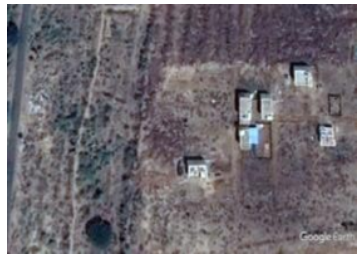

Figure 1. Input image of location (1a)

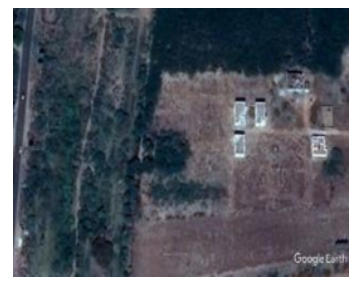

Figure2. Input image of location (1b)

\section{Otsu's Image Segmentation Method}

Otsu's method is utilized near achieve automatic image of thresholding. In the easiest structure, the algorithm restores a solitary intensity threshold that isolates into two classes of the pixels, foreground, and background. This threshold is controlled through limiting intra-class intensity variance, or identically, by expanding inter-class variance.The calculation carefully looks for the threshold that decreases the intra-class variance, characterized the weighted whole the variances between the two classes:

$\sigma_{\omega^{2}}\left(\mathrm{t}_{1}\right)=\omega_{1}\left(\mathrm{t}_{1}\right) \sigma_{0}{ }^{2}\left(\mathrm{t}_{1}\right)+\omega_{2}(\mathrm{t}) \sigma^{2}{ }_{1}\left(\mathrm{t}_{1}\right)(1)$

Weights $\omega 0$ and $\omega 1$ are the possibilities of the two classes divided through a threshold $t, \sigma^{2}{ }_{1}$ and $\sigma_{2}^{2}$ are variances of these two classes. For 2 classes, reducing the intra-class variance stands equivalent towards exploiting inter-class variance:

$$
\begin{aligned}
& \sigma^{2}{ }_{\mathrm{b}}\left(\mathrm{t}_{1}\right)=\sigma^{2}-\sigma^{2}{ }_{\omega}\left(\mathrm{t}_{1}\right)=\omega_{0}\left(\mu_{0}-\mu_{\mathrm{T}}\right)^{2}+\omega_{1}\left(\mu_{1}-\mu_{\mathrm{T}}\right)^{2} \\
& =\omega_{0}\left(\mathrm{t}_{1}\right) \omega_{1}\left(\mathrm{t}_{1}\right)\left[\mu_{0}\left(\mathrm{t}_{1}\right)-\mu_{1}\left(\mathrm{t}_{1}\right)\right]^{2}(2)
\end{aligned}
$$

The following relations can be easily verified: 
$\omega_{1} \mu_{1}+\omega_{2} \mu_{2}=\mu_{\mathrm{T}}$

$\omega_{1}+\omega_{2}=1(3)$

The class possibilities and class resources can be registered iteratively. These thought produces a success algorithm. Also, if the variances of the object and the background intensities are huge contrasted with the mean distinction or the image is seriously debated by added substance sound, the high-pitched gorgehistogram of the gray level is corrupted. Object segmentation (as well as Otsu's technique) are restricted by little article size, the small mean distinction among foreground and background pixels,enormous variance of the pixels that have a place with the object and those that have a place with the background, the huge measure of noise, etc.At every pixel, the normal gray-level estimation of the area is determined. Let the gray level of the given pixel alone separated into Ldiscrete qualities then the normal gray level is likewise separated into a similar L value. At that point, a couple is framed: the level of pixels and the normal of the area $(i, j)$. The all outnumber of events (recurrence), $\mathrm{f}_{\mathrm{ij}}$, the couple $(i, j)$, separated through the absolute number of pixels in an image $\mathrm{N}$, characterizes to cooperative likelihood mass function in ahistogram of 2-dimensional. Also, 2-dimensional Otsu's technique is created dependent on the 2-dimensional histogram.

\subsection{Pixel Intensity Clustering Algorithm}

The introduction of cluster centroids starts with a gauge cluster of weight. Cluster weight is the aggregate possibilities for a cluster in the pixel intensity. On the off chance that the info image is denoted to in $L$ pixel intensities $(0,1,2 \ldots L-1)$, the quantity of pixels at intensity level $i$ is indicated by $f_{i}$ and the all outnumber of pixels rises to $f=f_{0}+f_{1}+\ldots f_{l-1}$. The event likelihood $\mathrm{p}(i)$ pixel intensity to given is following by

$$
P_{(i)}=\frac{f_{i}}{f}, P(i) \geq 0, \sum_{i=0}^{L-1} P(i)=1
$$

The direct apportioning of a lot of picture pixel intensities is a helpful technique toward figure the primary cluster centroids. Rent $\mathrm{S}=\{\mathrm{e} 0$, e1... eQ-1 $\} \mathrm{Q} \leq \mathrm{L}$, be the arrangement of image pixel intensities with the end goal that for each component et $\epsilon$ $\mathrm{S}$ the condition $\mathrm{P}$ (et) $>0$ fulfilled. The reason for the linear apportioning technique is toward figure an underlying centroids of $\mathrm{K}$ cluster from the set $\mathrm{S}$. The variable $j$ is interpreted right now the cluster label, which is a number that partners anagreed image pixel intensity with its cluster. The pixel intensity is denoted $t, \mathrm{e}_{\mathrm{t} \epsilon \mathrm{S}}$ is attained as follows:

$$
\begin{gathered}
\omega_{\mathrm{j}}=\mathrm{P}\left(\mathrm{e}_{\mathrm{t}}\right) \\
t=(\operatorname{int})\left(i * \frac{Q}{k}\right), Q, k=2,3, \ldots \ldots . L
\end{gathered}
$$


The output image is made through consigning in the pixel intensity at the spatial area in the output image the cluster centroids for looking at pixel intensity at the spatial region in the input image. Given a pixel intensity at the area from the input image, the cluster label of this pixel intensity must be settled. The cluster is labelled,clusterfor centroid is that this pixel was circulated just before settled.

\section{Support Vector Machine}

In this work take an aerial images of urban settlements in the Tamil Nadu and is labeled as building and non-building classes. Every image in the image is RGB and has $1568 \times 1568$ pixels resolution where each pixel corresponds to a $3 \mathrm{~cm} \times 3 \mathrm{~cm}$ of Earth surface. The high-resolution images of earth surfaces is sliced the image into multiple small resolution $(256 \times 256)$ images with some pixels of overlap. These sliced images are used to train the model. So for an image, sliced are generated and the information about the location of the sliced image gets retained.In this work Linear SVM, Cubic SVM and Medium Gaussian SVM are consider for classification.SVM to train the image to classify the "Buildings" or "Non buildings". One technique for Linear SVM is the most up to date machine learning (data mining) algorithm to solving multiclass classification problems from the ultra-large data sets. The apparatuses a unique restrictive variant for a critical even algorithm for structuring a linear support vector machine. The equation of Linear SVM be able to be altered as follows:

$J 1(W 1, \xi)=1 / 2\|W\|^{2}+\mathrm{C} 1 \Sigma \xi_{j}$

Where $\mathrm{C} 1$ is a user-specified, positive, regularization parameter. In $£$ the inconstant is a directionhavingto all the sagging variables $\mathfrak{i}_{\mathrm{i}, \mathrm{i}}=1,2 \ldots$, 1.It very well may be deduced that Cubic SVM presents a higher accuracy in perceiving all the four feelings effectively. Additionally, Cubic SVM is favored because of its less calculation time which was $10 \mathrm{sec}$ for an element vector of measurement $12 * 1$ each for 100 examples. The SVM classifier is trained by shifting the length of the component vector. The testing procedure is executed for the building and non-building datasets independently.

\section{Experimental Results}

For an assessment of a created approach, a Google map image was utilized and the image is the input image. The information picture has segmented the object utilizing Otsu's method. An average gray-level estimation of the area is figured for every pixel. Accept that the gray level of any image is dividedkeen on $\mathrm{L}$ values and the average gray level is additionally isolated into similar L values. The subsequent (figure 4 and figure 5) bins of number are $L^{*} \mathrm{~L}$. The Resultant recurrence $f_{\mathrm{ij}}$, the couple $(\mathrm{i}, \mathrm{j})$ isolated through the complete number of pixels in an image N, gives the Mutual Possibility mass capacity histogram of 2-dimensional given as figure (3): 
This equation used to calculate the histogram value of grayscale image. $\mathrm{X}$-axis is denoted the value of intensity and Y-axis is denoted a pixel range $(256 * 256)$ in the image.
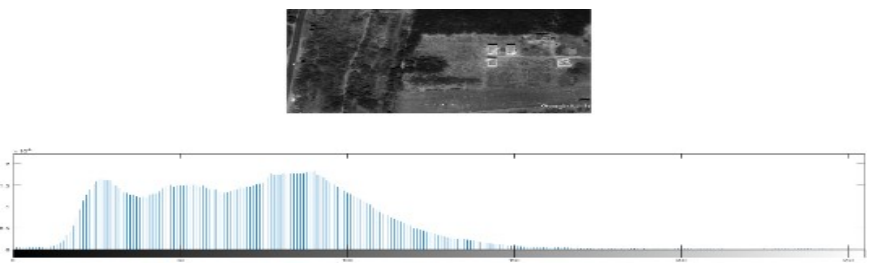

Figure 3. Histogram of Grayscale image.

Next locating the urban areas of the images figure (4 and 5), we first use the Pixel Intensity Clustering algorithm to detect buildings from the extracted urban areas, and then use the made rule to advance exclude some small errors in the primary building detection effects. The figure $4 \& 5$ are show that how many buildings of detected by our method.

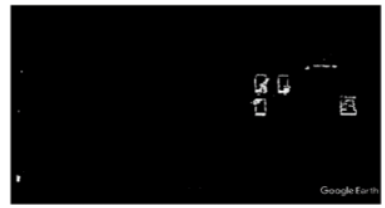

Figure 4. Objects identified location(1)

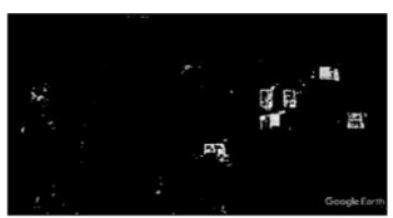

Figure 5. Objects identified location(2)

Finally the last step is classification of object identification image. For comparison purposes, an object-based classification of the same image has been classify the classification learner application. Classification Learner tool has used towards train the representations of these classifiers such as decision trees, discriminant analysis, support vector machines, logistic regression, nearest neighbors, naive Bayes, and ensemble classification.From the above classifier, we have used Linear SVM,Cubic SVM and Medium Gaussian SVM for predicting the classification accuracy. The accuracy evaluation results of comparision of Linear SVM,Cubic SVM and Medium Gaussian SVM are given in Table 1.

Table 1. Comparision of Linear SVM,Cubic SVM and Medium Gaussian SVM

\begin{tabular}{|l|l|l|l|}
\hline Parameters & Linear SVM & Cubic SVM & $\begin{array}{l}\text { Medium Gaussian } \\
\text { SVM }\end{array}$ \\
\hline Accuracy & $99.3 \%$ & $99.7 \%$ & $98.8 \%$ \\
\hline Kernel scale & Linear & Automatic & 40 \\
\hline Prediction Speed & $1400 \mathrm{obs} / \mathrm{sec}$ & $-1000 \mathrm{obs} / \mathrm{sec}$ & $-1100 \mathrm{obs} / \mathrm{sec}$ \\
\hline Training Speed & $23.64 \mathrm{sec}$ & $45.323 \mathrm{sec}$ & $64.617 \mathrm{sec}$ \\
\hline $\begin{array}{l}\text { ROC value(TPR } \\
\text { and FPR) }\end{array}$ & $0.93,1.00$ & $1.00,1.00$ & $0.53,0.97$ \\
\hline
\end{tabular}




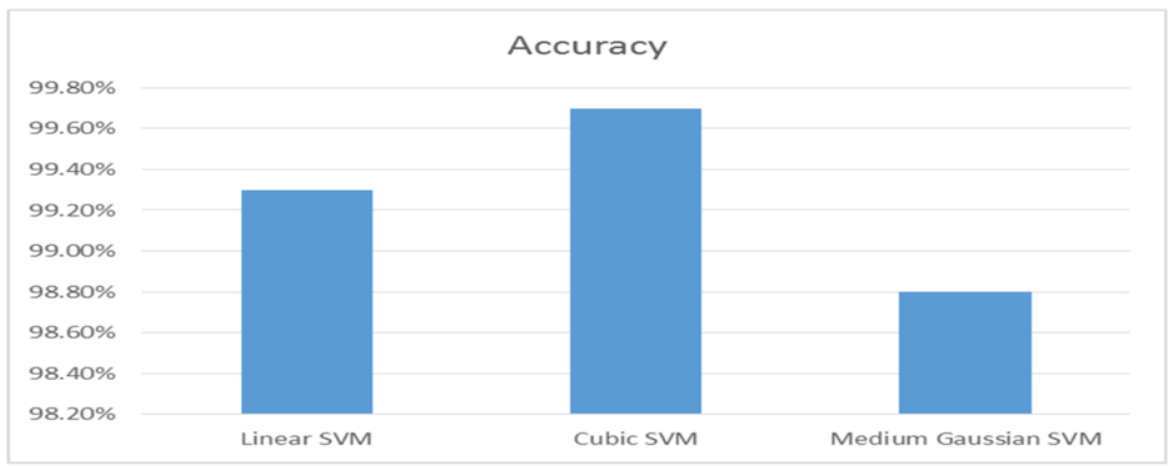

Figure 6. Accuracy values of three SVM's

The results of the validated model is accuracy value of three SVM's is given the figure 6 .

\section{Conclusion}

Overall, the three SVM grouping approach was discovered extremely encouraging for Object-Based Image Analysis. It has been demonstrated that it can deliver equivalent or surprisingly better outcomes than for supervised classification. The computational proficiency of three SVM was incredible, with just a couple of moments of runtime essential for training. This was hypothetically anticipated yet additionally, However, very large remote sensing datasets were tested. An awesome component of SVMs is that lone a small training set is expected to give generally excellent outcomes on the grounds that lone the support vectors are of significance during training.

\section{Acknowledgments}

The authors would like to thank BIT- Management for provided that support and materials related to educational research. Also, we are thankful to the Department of Science and Technology - Natural Resource Database Management System (DST NRDMS) by providing fund (Ref: NRDMS/01/181/2016 dated 13/02/2018).

\section{References}

[1] acihaliloglu I, Karta M. DCT and DWT based image compression in remote sensing images. In proceeding IEEE conference on antennas and propagation

[2] Ahmadi H, Nusrath A. Vegetation change detection of Neka River in Iran by using remote- sensing and GIS. Journal of geography and geology 2010; 2 (1),58-67.

[3] V.D.Ambeth Kumar, V.D.Ashok Kumar, S.Malathi and P.Jagaeedesh, "Intruder Identification using Footprint Recognition with PCA and SVM Classifiers" for the International Journal of Advanced Materials Research, 1345, 984-985 2014.

[4] Karaburun A. Estimation of $\mathrm{C}$ factor for soil erosion modeling using NDVI in Buyukcekmece watershed. Ozean journal of applied sciences 2010; 3,77-85.

[5] V. Wan and W. M. Campbell .Support vector machines for speaker verification and identification . in Proc. IEEE Workshop Neural Networks for Signal Processing, Sydney, Australia, Dec. 2000, pp. 775784. 
[6] E. Osuna, R. Freund, and F. Girosi .Training support vector machines: Application to face detection .in Proc. Computer Vision and Pattern Recognition, Puerto Rico, 1997, pp. 130-136.

[7] J. Platt .Fast training of support vector machines using sequential minimal optimization .in Advances in Kernel Methods: Support Vector Learning, B. Schölkopf, C. Burges, and A. J. Smola, Eds. Cambridge, MA: MIT Press, 1999, pp. 185-208.

[8] J. Roehrig, T. Doi, A. Hasegawa, B. Hunt, J. Marshall, H. Romsdahl, A. Schneider, R. Sharbaugh, and W. Zhang .Clinical results with R2 Imagechecker system . in Digital Mammography, N. Karssemeijer, M. Thijssen, J. Hendriks, and L. van Erning, Eds. Boston, MA: Kluwer Academic, 1998, pp. 395-400.

[9] N. Karssemeijer .A stochastic model for automated detection calcifications in digital mammograms .in Proc. 12th Int. Conf. Information Medical Imaging, Wye, U.K., July 1991, pp. 227-238. 